\section{Large-area laser-driven terahertz emitters}

\section{G. Klatt, D. Stephan, M. Beck, J. Demsar and T. Dekorsy}

Intense terahertz emitters are one of the most important components of terahertz $(\mathrm{THz})$ time-domain spectroscopy systems. In this presented report, the development of $\mathrm{THz}$ emitters over the last two decades is reviewed, and an outlook for future $\mathrm{THz}$ emitters is given. The physical principle behind the $\mathrm{THz}$ generation process is discussed for two types of emitters: state-of-the-art large-area photoconductive emitters are compared to $\mathrm{THz}$ emitters based on the photo-Dember effect. The latter do not require an external bias voltage. This passive character of the photo-Dember emitters has several advantages which are outlined.

The first photoconductive switch with electromagnetic waves in the picosecond regime was shown by Auston in 1984 [1]. The frequent employment of these 'Auston switches' then became established with the wide availability of femtosecond lasers such as the Kerr-lens modelocked titanium:sapphire in the 1990s. The most commonly used $\mathrm{THz}$ sources were subsequently based on either photoconductive switches [2] or the emission from semiconductor surfaces [3], in both cases irradiated with femtosecond laser beams. To date, these two methods introduced 20 years ago are still commonly employed for generating, by optical excitation, $\mathrm{THz}$ radiation in the frequency range $0.2-5 \mathrm{THz}$ $[4,5]$. Advances have also been made in the generation of $\mathrm{THz}$ radiation via optical rectification or difference frequency mixing $[6,7]$, but these will not be discussed further in this Letter.

Typically, the peak frequency of photoconductive sources or bare semiconductor surfaces lies around $1 \mathrm{THz}$, and the spectral coverage is limited to $3 \mathrm{THz}$. Drawbacks of these sources are the lack of longterm stability, scalability and the low conversion efficiencies from the optical to the THz regime. Further research led to the development of large-area photoconductive emitters [8-10], which exhibit one order of magnitude larger conversion efficiency compared to the first sources. Furthermore, recently it has been shown that, with an improved excitation geometry, conversion efficiencies up to $2 \times 10^{-3}$ are possible [11]. A new concept for the generation of intense $\mathrm{THz}$ radiation is based on the lateral photo-Dember effect [12]. Inspired by the large-area photoconductive emitters, these new emitters allow scalability, but do not require an external bias voltage.

The common feature of these $\mathrm{THz}$ generation methods is the emission of electromagnetic radiation from accelerated carriers in semiconductors such as gallium arsenide ( $\mathrm{GaAs}$ ) or indium gallium arsenide (InGaAs) [13]. Free carriers - electrons and holes - are generated at the surface of the semiconductor by femtosecond pulses with a photon energy above the gap energy of the semiconductor. For conventional photoconductive switches, electrodes deposited on the substrate material are externally biased and hence an electric field exists between the electrodes. The accelerating electric field $E_{e x t}$ is given by the externally applied voltage $U_{e x t}$ divided by the electrode spacing $d$. After photoexcitation, a time-dependent dipole oriented parallel to the surface builds up when the photogenerated electrons and holes are accelerated and separated in the electric field (see Fig. 1a). Owing to the higher mobility of the electrons compared to the holes, the electrons are mainly accelerated and contribute to the $\mathrm{THz}$ emission [14]. Owing to the dipole orientation, the generated $\mathrm{THz}$ radiation is then principally emitted in the forward and backward directions under normal incidence (see Fig. 1a)

A less common way to generate $\mathrm{THz}$ radiation is the photo-Dember effect $[14,15]$. This effect arises when electron-hole pairs are generated with a strong spatial gradient. In the simplest case, this occurs after optical excitation of a highly absorbing semiconductor surface which results in a carrier gradient perpendicular to the surface. Owing to different diffusion constants of electrons and holes, a space charge field $\mathrm{E}_{\text {Dember }}$ between the diffusing carrier distributions builds up. The photo-Dember field couples the diffusion of electrons and holes, and leads to ambipolar diffusion. Under pulsed excitation, this happens a short time after the initial generation process, and depends on the carrier gradient and the carrier densities [16, 17]. In the steady-state case, the photo-Dember field $\mathrm{E}_{\text {Dember }}$ can be estimated, under certain assumptions, from the drift-diffusion equation for electron and holes in combination with the Einstein relation [18]:

$$
E_{\text {Dember }}=\frac{k_{B} T}{e}\left(\frac{1}{n}\right)\left(\frac{\mu_{e}-\mu_{n}}{\mu_{e}+\mu_{n}}\right) \frac{d n}{d x}
$$

where $\mu_{e}$ and $\mu_{h}$ are the mobilities of electrons and holes, respectively. $n$ is the carrier density (the optically excited electron density is assumed to be equal to the hole density) and $x$ the spatial co-ordinate perpendicular to the surface. Experimentally the generation of $\mathrm{THz}$ radiation based on the photo-Dember effect has been observed for many different strongly absorbing semiconductors [17, 19-26]. The time-dependent dipole connected with the $\mathrm{THz}$ emission is oriented parallel to the optically excited carrier gradient and hence perpendicular to the excited surface. This is one of the drawbacks of $\mathrm{THz}$ generation by the photoDember effect - as well as for $\mathrm{THz}$ generation by surface field screening [27] - because for optimal excitation conditions at an angle of incidence of $45^{\circ}$, the out-coupled $\mathrm{THz}$ radiation is a factor of two smaller than for a dipole oriented parallel to the surface. Another limitation is the strength of the initial carrier gradient, which is given by the intrinsic absorption coefficient of the semiconductor at the excitation wavelength.
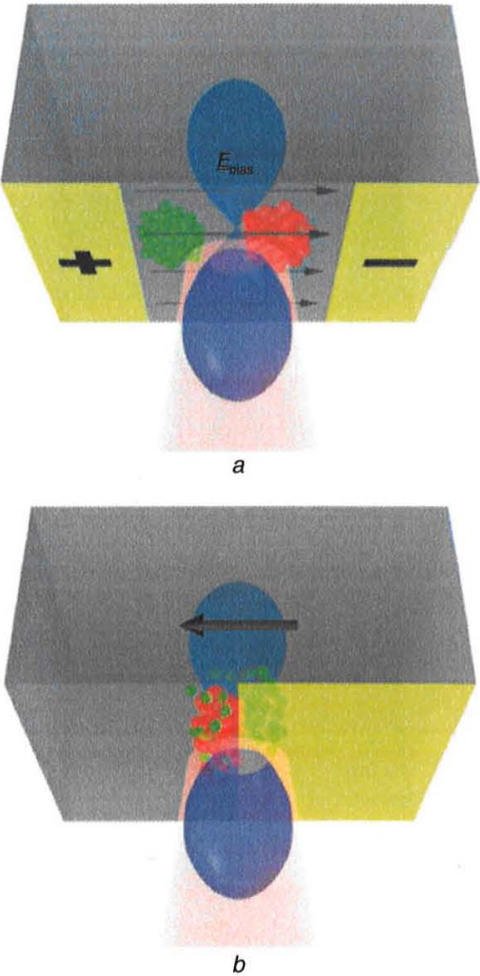

Fig. 1 Principle of $\mathrm{THz}$ emission from (Fig. Ia) a photoconductive switch and (Fig. Ib) lateral photo-Dember currents

In the case of a photoconductive switch, an external applied bias generates an electric field $\mathrm{E}_{\text {bias }}$ between the two electrodes. A femtosecond laser pulse (red cone) creates photoexcited carriers in the semiconductor grey. The electrons (green spheres) are accelerated towards the positive electrode, the holes (red spheres) towards the negative electrode. In contrast to the photoconductive switch, the photo-Dember emitters do not require external biasing. Here, photoexcitation of a partially metalised semiconductor surface with a femtosecond laser pulse results in a strong gradient in the carrier density at the edge of a metalised stripe. Since the photoinduced electrons diffuse much faster than the holes this gives rise to a photo-Dember field $\mathrm{E}_{\text {Dember }}$ perpendicular to the edge of the metalised stripe. In both pictures the blue lobes indicate the dipole radiation patterns of the arising $\mathrm{THz}$ radiation

To overcome these limitations the idea of lateral photo-Dember currents was suggested. In this case, the carrier gradient and therefore the time-dependent dipole is rotated by $90^{\circ}$, so that they are oriented parallel to the excited surface. This rotation can be easily achieved by partially shadowing the excitation area (see Fig. $1 b$ ). The strength of this gradient can be significantly higher than that obtained through the intrinsic absorption length of a semiconductor [12]. A proof of this simple design is given in Fig. $2 a$. The peak-to-peak amplitude of the emitted $\mathrm{THz}$ electric field is taken from a series of transients acquired at different positions relative to a metal edge underneath the laser spot. For details of the experimental setup we refer to $[12,28]$. Fig. $2 b$ shows the time transients when the laser spot is located at the metal-semiconductor edge. Depending on the edge (left or right) the time-domain data reveal a flipped single cycle electromagnetic emission indicating the change of the emitted $\mathrm{THz}$ electric field. Owing to the much higher electron 
mobility, the electrons diffuse faster underneath the metallic stripe than the holes, leading to the sign flip from the left to the right edge.
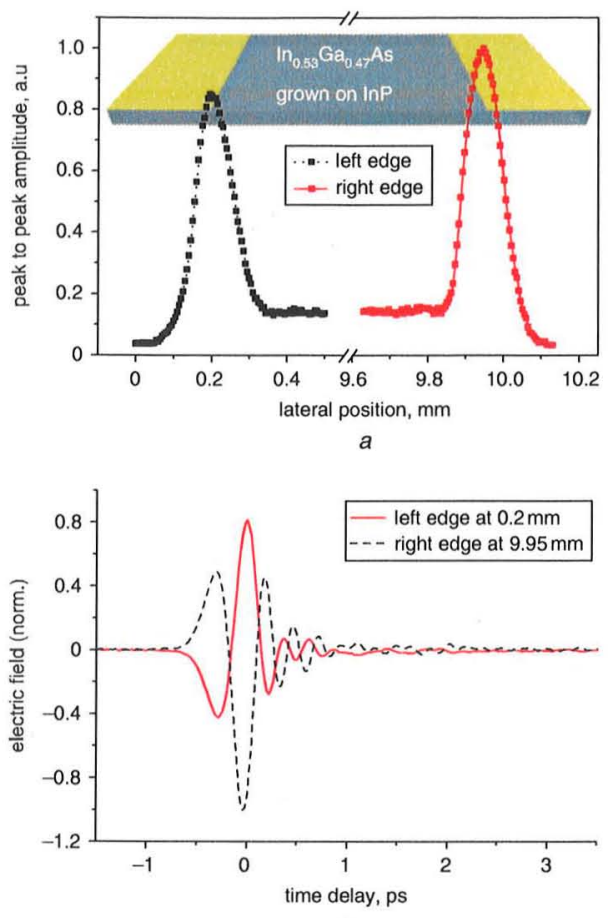

$b$

Fig. 2 Peak-to-peak amplitude of emitted THz electric field when metal semiconductor edge shown in background is moved underneath the laser spot. The line scans are normalised to the peak-to-peak amplitude at $9.95 \mathrm{~mm}$. (Fig. 2b) Fig. $2 b$ shows $\mathrm{THz}$ transients emitted from left and right metal-semiconductor edge when Gaussian-shaped optical excitation spot located exactly on metal edge. Left and right correspond to (Fig. 2a). The transients normalised to maximum electric field value at right edge

The orientation of the time-dependent dipole generating the $\mathrm{THz}$ radiation is an important factor for multiplexing the $\mathrm{THz}$ generation process and hence scaling up the active area. If the dipole is oriented parallel as in the case of the photoconductive switch and the lateral photoDember currents - multiplexing can be achieved by periodically adding emitter elements laterally (see Figs. $3 a$ and $b$ ). This is achieved by creating a metal-semiconductor-metal (MSM) structure processed by optical or electron beam lithography. For the photoconductive emitters, the electrode widths and spacings are chosen to be $5 \mu \mathrm{m}$. To avoid destructive interference of the $\mathrm{THz}$ radiation in the far field, it is important that every second gap between two electrodes - where the field is pointing in the opposite direction - is covered with an additional metallisation isolated from the electrodes [8]. This guarantees a coherent superposition of the $\mathrm{THz}$ radiation in the far field owing to unidirectional acceleration of the carriers over the entire active area (see Fig. $3 a$ ). The problem of high-voltages necessary for intense $\mathrm{THz}$ emission by single photoconductive switches is solved by keeping the gap dimensions small. In that case typical values for the acceleration field of the order of several tens of kilovolts per centimetre are easily obtained with low voltages. With these large-area photoconductive emitters of $10 \times$ $10 \mathrm{~mm}^{2}$ dimensions we demonstrated the generation of a peak electric field of the $\mathrm{THz}$ pulse in vacuum of $36 \mathrm{kV} / \mathrm{cm}$ when exciting the emitter with a pulse energy of $2.7 \mu \mathrm{J}[11]$.

An analogous concept for the multiplexing of photoconductive switches is also used for the photo-Dember emitters. The excitation of several periodic metal stripes would lead to destructive interference of the $\mathrm{THz}$ radiation in the far field, since the two carrier gradients of every stripe point in opposite directions. To achieve unidirectional carrier gradients and hence constructive interference in the far field, every second carrier gradient must be suppressed. Fig. $3 b$ shows one possibility to realise unidirectional carrier gradients experimentally, i.e. through wedged metal stripes. They generate strong carrier gradients at their thick opaque edge, while weak carrier gradients are obtained at the opposite side where the edges becoming thinner and transparent. The
Dember emitters discussed here are fabricated with a stripe width of about $2 \mu \mathrm{m}$ and a spacing of $1 \mu \mathrm{m}$ and have a size of $1 \times 1 \mathrm{~mm}^{2}$. At the moment the size is limited by the fabrication process.

A comparison of the multiplexed emitters was performed in a rapidscanning $\mathrm{THz}$ precision spectrometer based on asynchronous optical sampling, as shown in Fig. 4 [28, 29]. At a repetition rate of the femtosecond laser of $1 \mathrm{GHz}$ the pulse energies are small (less than $1 \mathrm{~nJ}$ ), so that the excitation spot can be set to $55 \mu \mathrm{m}$ full-width-half-maximum without running into saturation effects. The photo-Dember emitter is manufactured on the same $\mathrm{In}_{0.53} \mathrm{Ga}_{0.47} \mathrm{As}$ substrate as the single metalsemiconductor edge shown in Fig. $2 a$ and shows a four times larger $\mathrm{THz}$ electric field as the single edge. The multiplexed photo-Dember emitters are superior to the large-area photoconductive emitters when the latter is being biased with an acceleration field below $10 \mathrm{kV} / \mathrm{cm}$ or less (see Fig. 4). Besides the higher electric field, the multiplexed photo-Dember emitter also shows a broader spectrum and higher peak frequency than the large-area photoconductive emitter (see inset of Fig. 4).
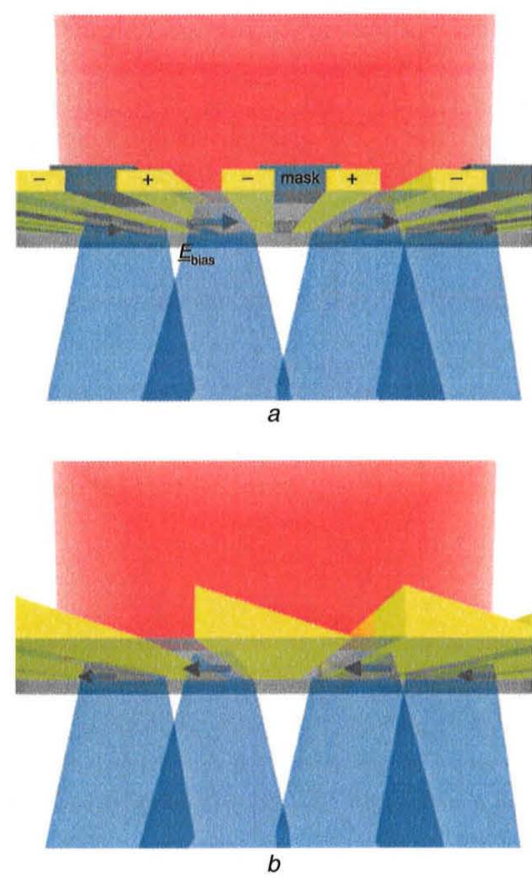

Fig. 3 Sketch of multiplexed THz emitters based on principle of (Fig. 3a) a photoconductive switch and (Fig. 3b) lateral photo-Dember currents

In the case of the photoconductive emitter every second gap between the electrodes is masked to avoid destructive interference of the generated $\mathrm{THz}$ radiation (indicated by blue areas) in the far field. Analagously, for the photo-Dember emitters, every second carrier gradient is suppressed by wedged metal stripes to achieve unidirectional carrier gradients

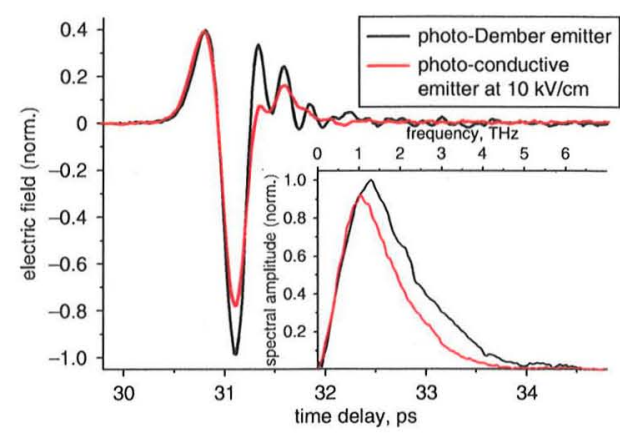

Fig. 4 Comparison of time transients and Fourier spectra (inset) of large area photoconductive emitter operating at $10 \mathrm{kV} / \mathrm{cm}$ bias electric field and multiplexed photo-Dember emitter operating without bias voltage Both graphs normalised to corresponding maximum value of photo-Dember emitter

In conclusion, we have discussed two state-of-the-art $\mathrm{THz}$ sources, i.e. interdigitated photoconductive emitters, and emitters based on the 
lateral photo-Dember effect. Multiplexed photo-Dember emitters show a comparable performance to large area photoconductive emitters. Owing to their passive character they are robust against local damage, e.g. shortcircuit problems. Also, these emitters do not suffer from substrate or technology related problems leading to high dark currents and associated heating of emitters as in the photoconductive case. The concept of photo-Dember emitters is not limited to certain substrates; hence they are also suitable for excitation with femtosecond fibre lasers.

Acknowledgments: We wish to thank F. Hilser, H. Schäfer, K. Huska, G. Bastian, U. Lemmer, M. Fischer, J. Faist, A. Dreyhaupt, S. Winnerl, and $\mathrm{M}$. Helm for their contribution to the development of high-efficiency $\mathrm{THz}$ emitters. This work is supported by the Baden-Württemberg Stiftung, the Ministry of Science, Research and the Arts of BadenWürttemberg and the Deutsche Forschungsgemeinschaft. J. Demsar acknowledges the Alexander-von-Humboldt Stifung.

doi: $10.1049 / \mathrm{el} .2010 .3315$

G. Klatt, D. Stephan, M. Beck, J. Demsar and T. Dekorsy (Department of Physics and Center for Applied Photonics, University of Konstanz, 78457 Konstanz, Germany)

E-mail: Thomas.Dekorsy@uni-konstanz.de

\section{References}

1 Auston, D.H., Cheung, K.P., and Smith, P.R.: 'Picosecond photoconducting Hertzian dipoles', Appl. Phys. Lett., 1984, 45, pp. 284-286

2 Fattinger, C., and Grischkowsky, D.: 'Terahertz beams', Appl. Phys. Lett., 1989, 54, pp. 490-492

3 Zhang, X-C., Hu, B.B., Darrow, J T, and Auston, D.H. 'Generation of femtosecond electromagnetic pulses from semiconductor surfaces', Appl. Phys. Lett., 1990, 56, pp. 1011-1013

4 Planken, P.C.M., Rijmenam, C.E.W.M.van, and Schouten, R.N.: 'Optoelectronic pulsed THz systems', Semicond. Sci. Technol, 2005, 20, S121-S127

5 Tonouchi, M.: 'Cutting-edge terahertz technology', Nature Photonics, 2007, 1, pp. 97-105

6 Hebling, J., Almasi, G., Kozma, I., and Kuhl, J.: 'Velocity matching by pulse front tilting for large area THz-pulse generation', Opt. Express, 2002, 10, pp. $1161-1166$

7 Sell, A., Leitenstorfer, A., and Huber, R.: 'Phase-locked generation and field-resolved detection of widely tunable terahertz pulses with amplitudes exceeding $100 \mathrm{MV} / \mathrm{cm}^{3}$, Opt. Lett., 2008, 33 pp. 2767-2769

8 Dreyhaupt, A., Winnerl, S., Dekorsy, T., and Helm, M.: 'High-intensity terahertz radiation from a microstructured large-area photoconductor', Appl. Phys. Lett., 2005, 86, p. 121114-3

9 Acuna, G., Buersgens, F., Lang, C., Handloser, M., Guggenmos, A., and Kersting, R.: 'Interdigitated terahertz emitters', Electron. Lett. 2008,44 , pp. $229-231$
10 Awad, M., Nagel, M., Kurz, H., Herfort, J., and Ploog, K.: "Characterization of low temperature GaAs antenna array terahertz cmitters', Appl. Phys. Lett, 2007, 91, p. 181124

11 Beck, M., Schäfer, H., Klatt, G., Demsar, J., Winnerl, S., Helm, M., and Dekorsy, T.: 'Impulsive terahertz radiation with high electric fields from an amplifier-driven large-area photoconductive antenna', Opt. Express, 2010, 18, pp. $9251-9257$

12 Klatt, G., Hilser, F., Qiao, W., Beck, M., Gebs, R, Bartels, A., Huska, K., Lemmer, U., Bastian, G., Johnston, M., Fischer, M., Faist, J., and Dekorsy, T.: 'Terahertz emission from lateral photo-Dember currents', Opt. Express, 2010, 18, pp. 4939-4947

13 Shan, J., and Heinz, T.: 'Terahertz radiation from semiconductors', Vol. 92 of Ultrafast Dynamical Processes in Semiconductors, Top. Appl. Phys. (Springer, Berlin/Heidelberg, 2004)

14 Demsar, J, and Dekorsy, T.: 'Carrier dynamics in bulk semiconductors and metals after ultrashort pulse excitation', Opt. Tech. Solid-State Mater. Characteriz. (Francis \& Taylor, New York, in print, 2010)

15 Dember, H.: 'Über eine photoclektronische Kraft in KupferoxydulKristallen', Zeitschrift für Physik, 1931, 32, p. 554

16 Dekorsy, T., Pfeifer, T., Kütt, W., and Kurz, H.: 'Subpicosecond carricr transport in GaAs surface-space-charge fields', Phys. Rev. B, 1993, 47, pp. $3842-3849$

17 Dekorsy, T., Auer, H., Bakker, H.J., Roskos, H.G., and Kurz, H.: 'THz electromagnetic emission by coherent infrared-active phonons', Phys. Rev. $B, 1996,53$, pp. $4005-4014$

18 Seeger, K.: 'Semiconductor physics - an introduction' (Springer, Berlin, 2004, 9th edn)

19 Gu, P., Tani, M., Kono, S., Sakai, K., and Zhang, X.-C.: 'Study of terahertz radiation from InAs and InSb', J. Appl. Phys., 2002, 91, pp. 5533-5537

20 Heyman, J.N., Coates, N., Reinhardt, A., and Strasser, G.: 'Diffusion and drift in terahertz emission at GaAs surfaces', Appl. Phys. Lett., 2003, 83, pp, 5476-5478

21 Hasselbeck, M.P., Schlie, L.A., and Stalnaker, D.: 'Emission of electromagnetic radiation by coherent vibrational waves in stimulated Raman scattering', Appl. Phys. Lett., 2004, 85, pp. 173-175

22 Nakajima, M., Oda, Y., and Suemoto, T.: 'Competing terahertz radiation mechanisms in semi-insulating $\operatorname{InP}$ at high-density excitation', Appl. Phys. Lett., 2004, 85, pp. 2694-2696

23 Ascázubi, R., Shneider, C., Wilke, 1., Pino, R., and Dutfa, P.S. 'Enhanced terahertz emission from impurity compensated GaSb', Phys. Rev. B, 2005, 72, p. 045328

24 Urbanowicz, A., Adomavicius, R., and Krotkus, A.: 'Terahertz emission from photoexcited surfaces of Ge crystals', Physica B, 2005, 367, pp. $152-157$

25 Ascázubi, R, Wilke, I, Kim, KJ, and Dutta, P. 'Terahertz emission from $\mathrm{Ga}_{1-\mathrm{x}} \mathrm{In}_{\mathrm{x}} \mathrm{Sb}$, Phys. Rev. B, 2006, 74, p. 075323

26 Que, C.T., Edamura, T., Nakajima, M., Tani, M., and Hangyo, M.: 'Terahertz radiation from InAs films on silicon substrates excited by femtosecond laser pulses', Jpn. J. Appl. Phys., 2009, 48, p. 010211

27 Zhang, X.-C., and Auston, D.H.: 'Optoelectronic measurement of semiconductor surfaces and interfaces with femtosecond optics', J. Appl. Phys., 1992, 71, pp. 326-338

28 Bartels, A., Cerna, R., Kistner, C., Thoma, A., Hudert, F., Janke, C., and Dekorsy, T.: 'Ultrafast time-domain spectroscopy based on high-speed asynchronous optical sampling', Rev. Sci. Instrum., 2007, 78, p. 035107

29 Klatt, G., Gebs, R., Janke, C., Dekorsy, T., and Bartels, A.: 'Rapidscanning terahertz precision spectrometer with more than $6 \mathrm{THz}$ spectral coverage', Opt. Express, 2009, 17, pp. 22847-22854 